\title{
Large-scale secondary structures in duct flow
}

\author{
By B. GALLETTI AND A. BOTTARO ${ }^{2}$ \\ ${ }^{1}$ DIASP, Politecnico di Torino, Corso Duca degli Abruzzi 24, 10129 Torino, Italy \\ ${ }^{2}$ DIAM, Università di Genova, Via Montallegro 1, 16145, Genova, Italy
}

(Received 12 March 2004 and in revised form 5 May 2004)

The spatial growth of small perturbations developing on a fully developed base flow in a duct with two inhomogeneous cross-flow directions is examined. For a laminar mean flow it is shown that optimally configured vortices at an upstream cross-section induce large transient amplification of a disturbance energy norm downstream. Such a linear growth is a likely initial stage of transition in ducts for which the cross-section is square or of moderate aspect ratio, asymptotically stable according to conventional eigen-analyis. With the increase of the cross-sectional aspect ratio the transient growth results of the plane channel flow case are approached. The optimization methodology is then employed to study the transient amplification of large-scale secondary structures developing in a square duct for which the unidirectional base motion has a turbulent-like profile. Under the hypothesis that fine-scale turbulence does not affect the growth of large-scale secondary flows, it is shown that cross-stream vortices are sustained by the mean flow and grow maximally over a streamwise distance of several thousand viscous units of length.

\section{Introduction}

The motion of fluids in ducts of rectangular cross-section is of theoretical and practical interest, and its understanding has motivated much research in the last eighty years. The observations that Nikuradse (1926) made in the course of his doctoral studies in Göttingen indicate that, above some value of the Reynolds number, secondary flows appear near the corners of the duct cross-section, leading to a deformation of the mean velocity contours. Such corner vortices have been classified as secondary flows of Prandtl's second kind, and ascribed to the presence of turbulence, as opposed to the secondary vortices of the first kind which arise from the skewing of the cross-stream vorticity, and for which secondary velocities are typically one order of magnitude larger.

Significant experimental contributions to the study of corner vortices arising in ducts of square cross-section have been published by Brundrett \& Baines (1964) and Gessner (1973). The former observed that the vortices penetrate farther into the corners with the increase of the Reynolds number, and ascribed the secondary flows to the gradients of the normal Reynolds stresses. Gessner (1973), on the other hand, suggested that the secondary flows are produced by the transverse gradients of the primary shear stresses occurring in the corner region. The results by Gessner were surprising since they played down the role of the anisotropy of the turbulent normal stresses. At the same time, Launder \& Ying (1973) had shown that a turbulence model which used an isotropic eddy viscosity was incapable of capturing corner vortices, thus highlighting the importance of anisotropic modelling. Several studies followed, aimed at showing that through appropriate anisotropic modelling of the Reynolds 
stresses, corner vortices could be captured more or less correctly. One notable such work is that by Demuren \& Rodi (1984); they solved a spatially parabolic set of equations for the mean flow, closing the turbulence problem with an algebraic stress model, using transport equations for the turbulent kinetic energy and the dissipation rate. The numerical results reproduced many features of the cross-stream motion in a turbulent square duct, thus, apparently, settling the issue. However, more recent attempts (Mompean et al. 1996; Mompean 1998) at predicting corner vortices using a variety of turbulence closure strategies have consistently underestimated the magnitudes of the secondary flows compared to direct simulation results for the same parametric conditions (Gavrilakis 1992). The issue of the origin of the corner vortices is still open as noted by Gavrilakis who concluded that " $a$ theory on the flow structures that give rise to the observed mean flow is not yet available". With the objective of making progress towards such a theory, we start from the hypothesis that large-scale secondary vortices in square and rectangular ducts respond linearly to the mean shear, over a large range of Reynolds numbers and flow regimes.

We begin by studying the initial amplification of disturbances in the laminar squareduct case and demonstrate that - despite the fact that all eigenmodes of the linearized problem predict asymptotic stability (Tatsumi \& Yoshimura 1990) - cross-stream flows can appear in the early transitional regime, associated with large (order $R e^{2}$ ) disturbance energy growth. The study is then extended to the case of arbitrary-aspect-ratio ducts. Like pipe and plane channel flows, the results suggest that the initial algebraic growth of perturbations has an important role to play in the transition process.

Then, rather than attempting a nonlinear study of the development of optimal disturbances (i.e. those disturbances which evoke the largest growth), we focus on the dynamics of perturbations developing over a fully-developed turbulent flow profile. The underlying rationale for this approach stems from a number of studies, starting from Crighton \& Gaster (1976) who considered a turbulent jet and argued that turbulence sets up an equivalent laminar flow profile for large-scale coherent modes. Agreement between linear stability modes (frequency and spatial scales) and experimental measurements supported the linear hypothesis. Along similar lines, Landahl (1990) proposed a simple model for the bursting phenomenon in turbulent boundary layers, based on the inflectional instability of growing streaks, whose physical realism prompted him to state that "the success of a quasi-laminar model like the present one in explaining some of the behaviour of the coherent structures in the turbulent boundary layer suggests the possibility that certain features of turbulent flow may be studied in a laminar environment".

In the present configuration the optimal linear growth of small disturbances results in the formation of corner vortices in the cross-section, thus supporting the hypothesis that the presence of secondary flows is a consequence of the linear deterministic evolution of the flow. This is in line with the arguments by Gessner (1973) on the role of the turbulent stresses. The importance of the anisotropic modelling of the Reynolds stresses cannot, clearly, be overstated: it is needed for a proper description of the turbulent field and to account correctly for the spectral distribution of energy once fluctuations have attained large amplitudes.

\section{The algebraic growth of disturbances in the laminar regime}

We consider an infinitely long duct of cross-sectional aspect ratio $A$ filled with an incompressible fluid whose motion along the $x$-axis is fully developed. The shape of the laminar base flow $U(y, z)$ is given, for example, in Tatsumi \& Yoshimura (1990). They studied the exponential damping/amplification of small perturbations in time 
numerically focusing on Tollmien-Schlichting-like waves and concluded that as the aspect ratio $A$ increases, the critical Reynolds number decreases (tending towards the value of 5772 of the plane channel case), and the critical wavenumber increases (the limiting plane-channel value is 1.02). For example, for $A=8$ it is reported that $R e_{c}=6800$ and $\alpha_{c}=0.98$. Furthermore, they indicated that for $A$ less than roughly 3.2 the flow is linearly stable. Comparison with experiments (Kao \& Park 1970) conducted with $A=8$ revealed very poor agreement, the measured value of the critical Reynolds number being close to 1200 with a corresponding wavenumber close to 0.4 . Tatsumi \& Yoshimura (1990) ascribed this disappointing comparison to the effect of nonlinearities in the experiments.

The experiments by Kao \& Park (1970) were meant to produce streamwise travelling waves, and were conducted both with and without controlled excitation. The authors report that: (i) the value of the Reynolds number at which the laminar flow breaks down is practically the same with or without artificial excitation; (ii) the unstable region includes the zero-frequency, zero-streamwise-wavenumber range; (iii) the disturbances are three-dimensional. Assuming that the experiments were conducted in a noisy environment $\uparrow$ the findings reported can be reconciled with the theory of by-pass transition (Schmid \& Henningson 2001), according to which environmental three-dimensional disturbances are initially amplified in a transient manner, until perturbation amplitude levels are attained for which nonlinear effects, parametric resonances and/or secondary instabilities take over to complete the transition process. Thus, the study of the transient growth in space of disturbances is necessary to assess the importance of this mechanism and to establish it as a viable initial path to transition in rectangular duct flow.

\subsection{Transient growth: the theory}

The initial phase of algebraic growth of streamwise-elongated secondary structures superposed onto a parallel base flow $U(y, z)$ can be adequately described by the use of linearized equations in which a long scale is used to normalize streamwise lengths and a short scale for the cross-stream directions. The plane duct is bounded by four solid walls at $y= \pm A h$ and $z= \pm h$; by scaling the cross-stream axes $(y, z)$ with $h$, and the streamwise coordinate $x$ with $h / \epsilon, \epsilon$ a small parameter, it follows that $U_{0}$, maximum velocity in the channel, should be used as scale for the streamwise perturbation velocity $u$, together with $\epsilon U_{0}$ for the cross-stream components $(v, w)$. Pressure is normalized by $\rho\left(\epsilon U_{0}\right)^{2}$, with $\rho$ the density of the fluid. Steady equations are considered because of the low-frequency behaviour of streaks reported by several experimental observations, e.g. Matsubara \& Alfredsson (2001), and of the result by Luchini (2000) that stationary streamwise vortices at the inflow produce the largest transient growth in a boundary layer. The scaling employed here yields $\epsilon=R e^{-1}=v U_{0}^{-1} h^{-1}$ and simplifies the equations at leading order (in particular it renders them parabolic in $x$ ), but has no effect on the results for optimal perturbations (Biau \& Bottaro 2004). The Reynolds-number-independent, dimensionless equations are:

$$
\left.\begin{array}{l}
u_{x}=-v_{y}-w_{z}, \\
U u_{x}=-U_{y} v-U_{z} w+\Delta_{2} u, \quad U v_{x}=-p_{y}+\Delta_{2} v, \quad U w_{x}=-p_{z}+\Delta_{2} w,
\end{array}\right\}
$$

$\dagger$ No mention is made in the paper of the prevailing receptivity conditions; the authors cite fine mesh screens placed ahead of the inlet section of the channel, but do not provide values of the turbulence level at the inflow. 
with the operator $\Delta_{2}=\partial_{y y}+\partial_{z z}$, together with no-slip conditions at the solid walls. An energy-like norm is defined as

$$
E(x)=\int_{-1}^{+1} \int_{-A}^{+A}\left[u^{2}+\left(v^{2}+w^{2}\right) / R e^{2}\right] \mathrm{d} y \mathrm{~d} z .
$$

The presence of the Reynolds number in the definition of the norm is due to the velocity scales adopted. The goal of the analysis is to find the initial condition which maximizes a gain $G$, a function of the distance $x$ over which the extremum is sought, defined as $G(x)=E(x) / E(0)$. Clearly such a ratio is a maximum, and is $O\left(R e^{2}\right)$, when $u(0, y, z)=0$, so that the objective functional to leading order reduces to

$$
G(x)=\operatorname{Re}^{2} \frac{\iint u(x, y, z)^{2} \mathrm{~d} y \mathrm{~d} z}{\iint\left[v(0, y, z)^{2}+w(0, y, z)^{2}\right] \mathrm{d} y \mathrm{~d} z} .
$$

The theory of optimal disturbances appeared around fifteen years ago, thanks to the pioneering efforts of Farrell (1988). In the first studies on optimals the initial disturbance was sought by considering it as a weighted sum of a truncated set of eigenfunctions of the corresponding eigen-problem. In the present case, this would mean taking disturbances of the form exp(i $\alpha x)$ and computing numerically a sufficient number of eigenmodes, so as to adequately represent an arbitrary initial condition. It is however extremely difficult to resolve well a sufficiently large number of modes of this two-dimensional eigen-problem to build an adequate basis (Robitaillié-Montané \& Casalis 2003). Hence, a different strategy - borrowed from optimal control theory has been used, based on the repeated numerical resolution of the direct parabolic problem and its adjoint.

The parabolic equations (2.1) are treated with a pseudospectral collocation method in the $(y, z)$-plane, coupled with a finite difference scheme along the $x$-axis. The unknowns are the values of the primitive variables $p, u, v$ and $w$ at the $N_{y} \times N_{z}$ Gauss-Lobatto grid points $y_{i}=A \cos \pi(i-1) /\left(N_{y}-1\right)$ with $i=1, \ldots, N_{y}$ and $z_{j}=\cos \pi(j-1) /\left(N_{z}-1\right)$ with $j=1, \ldots, N_{z}$. For instance, if $u_{i j}$ denotes the value of $u$ at the point $\left(y_{i}, z_{j}\right)$ the approximation $u(y, z) \approx \sum_{i=1}^{N_{y}} \sum_{j=1}^{N_{z}} \phi_{i}(y / A) \phi_{j}(z) u_{i j}$ is used, where $\phi_{i}(y / A)$ and $\phi_{j}(z)$ are the Lagrangian interpolating polynomials based on the nodes $y_{i}$ and $z_{j}$, respectively. By denoting as $\boldsymbol{p}, \boldsymbol{u}, \boldsymbol{v}$ and $\boldsymbol{w}$ the column vectors containing the interior nodal values of the variables, the discretized equations (2.1) become

$$
\boldsymbol{Q q}_{x}=\boldsymbol{R} \boldsymbol{q}
$$

where $\boldsymbol{q}=\left[\begin{array}{llll}\boldsymbol{p} & \boldsymbol{u} & \boldsymbol{v} & \boldsymbol{w}\end{array}\right]^{T}$ is a $4 M \times 1$ vector, $\boldsymbol{Q}$ and $\boldsymbol{R}$ are $4 M \times 4 M$ matrices, easy to recover from (2.1), and $M=\left(N_{y}-2\right)\left(N_{z}-2\right)$ is the total number of interior nodes. The no-slip boundary conditions are enforced implicitly. Since the matrix $\boldsymbol{Q}$ is singular, we adopt an implicit finite difference discretization of (2.4) along the $x$-axis. The nodal values of the pressure at $x=0$ can be chosen arbitrarily, so that the initial state vector is set equal to $\boldsymbol{q}^{(0)}=\left[\begin{array}{lll}\mathbf{0} \mathbf{0} & \boldsymbol{v}(0) & \boldsymbol{w}(0)\end{array}\right]^{T}$, with an arbitrary initial guess for $\boldsymbol{v}(0)(\boldsymbol{w}(0)$ follows from the equations). Thus, the discretized problem consists of the following recurrence relations:

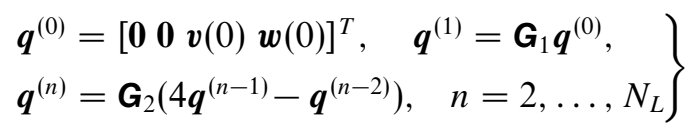


where $\boldsymbol{q}^{(n)}=\boldsymbol{q}(n \Delta x), \mathbf{G}_{1}=(\boldsymbol{Q}-\boldsymbol{R} \Delta x)^{-1} \boldsymbol{Q}, \mathbf{G}_{2}=(3 \boldsymbol{Q}-2 \boldsymbol{R} \Delta x)^{-1} \boldsymbol{Q}$, and $L=N_{L} \Delta x$ is the length of the channel. The Gauss-Legendre quadrature formula gives the gain

$$
G(L)=R e^{2} \frac{\boldsymbol{u}(L)^{T} \boldsymbol{H} \boldsymbol{u}(L)}{\boldsymbol{v}(0)^{T} \boldsymbol{H} \boldsymbol{v}(0)+\boldsymbol{w}(0)^{T} \boldsymbol{H} \boldsymbol{w}(0)},
$$

with $\boldsymbol{H}$ a symmetric $4 M \times 4 M$ matrix (Canuto et al. 1988). The optimization problem consists in finding the $v(0)$ that maximize $G(L)$ under the constraint given by (2.4). This is equivalent to the unconstrained maximization of the Lagrangian functional

$$
\mathscr{L}(\boldsymbol{v}(0), \boldsymbol{q}, \boldsymbol{r})=G(L)+\int_{0}^{L} \boldsymbol{r}^{T}\left(\boldsymbol{Q} \boldsymbol{q}_{x}-\boldsymbol{R} \boldsymbol{q}\right) \mathrm{d} x,
$$

where the $4 M \times 1$ vector $\boldsymbol{r}=\left[\begin{array}{llll}\boldsymbol{a} & \boldsymbol{b} & \boldsymbol{c} & \boldsymbol{d}\end{array}\right]^{T}$ is a Lagrange multiplier. By imposing stationarity of $\mathscr{L}$ with respect to $\boldsymbol{q}$ the following discrete adjoint equation is obtained:

$$
\mathbf{Q}^{T} \boldsymbol{r}_{x}=-\boldsymbol{R}^{T} \boldsymbol{r}
$$

(which is different from the discretized version of the system adjoint to (2.1)), plus

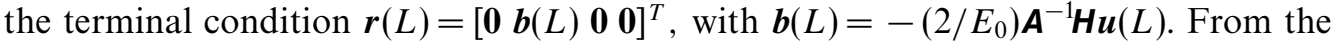
stationarity of $\mathscr{L}$ with respect to $\boldsymbol{v}(0)$ (the 'control') the optimality condition follows:

$$
\boldsymbol{v}(0)=-\frac{E_{0}^{2}}{2 E_{f}} \boldsymbol{H}^{-1} \boldsymbol{A} \boldsymbol{c}(0),
$$

with $\boldsymbol{A}$ the diagonal matrix having on the main diagonal the values of the base flow $U(y, z)$ at the interior nodes. The discrete adjoint equation proceeds from downstream to upstream:

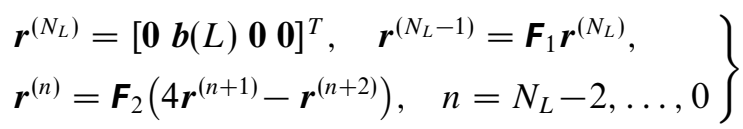

where $\boldsymbol{r}^{(n)}=\boldsymbol{r}(n \Delta x), \boldsymbol{F}_{1}=\left(\boldsymbol{Q}^{T}-\boldsymbol{R}^{T} \Delta x\right)^{-1} \boldsymbol{Q}^{T}, \boldsymbol{F}_{2}=\left(3 \boldsymbol{Q}^{T}-2 \boldsymbol{R}^{T} \Delta x\right)^{-1} \boldsymbol{Q}^{T}$. In practice, the direct problem (2.5) is iterated until the value of $\boldsymbol{b}(L)$ is found; this value is used to initialize the adjoint solver (2.7). Backward integration of the adjoint equations gives $\boldsymbol{c}(0)$ and we can thus update $\boldsymbol{v}(0)$ through (2.6). After a few direct-adjoint iterations (ranging from five when $A$ is large to fifteen for $A=1$ ) the maximum gain is typically found, for the chosen length $L$. To find the optimal perturbation the procedure is repeated for several values of $L$, until the largest gain $G$ is found. The corresponding length is denoted $x_{o p t}$.

Figure 1(c) displays the largest attainable gain against the streamwise distance $x$, for some values of $A$. For the square $\operatorname{duct}(A=1)$ the optimal value of $G$ is relatively low, a consequence of the fact that for this geometry viscous diffusion from the sidewalls acts most efficiently. As $A$ increases the gain attains a maximum over longer distances, approaching asymptotically the curve for the plane channel flow (Biau \& Bottaro 2004). The optimal gain and the corresponding $x_{\text {opt }}$ are reported in figure $1(a, b)$ as functions of the aspect ratio. For $A=1$ both maximum gain and corresponding $x_{\text {opt }}$ are very close to the values of pipe Poiseuille flow, provided by Reshotko \& Tumin (2001). In the square duct two vortices disposed obliquely in the cross-section constitute the optimal perturbation at $x=0$; their downstream development yields two large streaks of high and low streamwise momentum. This result, displayed in figure 2(top row), closely resembles the optimal disturbance found in pipe Poiseuille flow by Reshotko \& Tumin (2001), for which the azimuthal wavenumber is $m=1$. With the increase of the cross-sectional aspect ratio $A$ the results approach progressively 

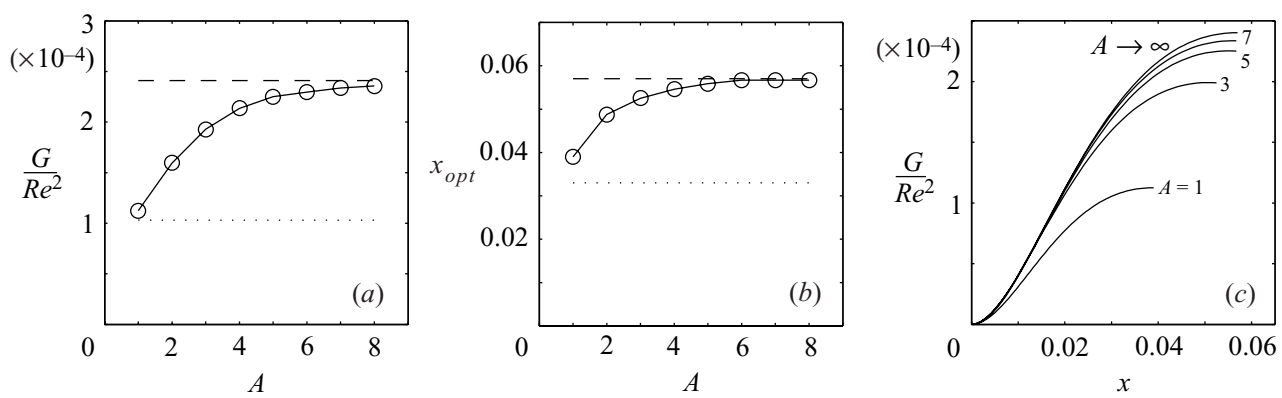

FIGURE 1. Evolution of the gain with $x$, for some values of $A(c)$. Maximum gain $(a)$ and corresponding position $(b)$ as function of $A$; the upper dashed lines correspond to the asymptotic results for $A \rightarrow \infty$, the dotted lines are the results for pipe flow (Reshotko \& Tumin 2001).

those of the plane channel case (cf. figure 2). For $A=8$ the disturbance fields near the centre of the cross-section are identical - to graphical accuracy - to those in the plane channel. It is important to stress that, as indicated by Luchini (2000), the procedure adopted is equivalent to performing power iterations to identify the largest singular value of the operator propagating the initial condition downstream. The singular values are well separated from one another in the spectrum - as attested by the rapid convergence of the procedure to the maximum gain, particularly for large values of $A$ - and this has the consequence that almost any inflow condition produces the same downstream pattern (provided the initial guess at $x=0$ is not exactly orthogonal to the optimal perturbation). The second and third singular values can be computed by forcing the initial condition to be always orthogonal to the first (or the first and the second) singular function(s). Such solutions are shown in figure 3 for the case $A=1$ and correspond, respectively, to four and eight vortices/streaks; these flows could prevail and be observed over short streamwise stretches, depending on the receptivity conditions. Indeed, the results of optimization procedures carried out over streamwise distances smaller than the optimal distance reveal that (relative) maximum gains are associated with states characterized by four transverse vortices.

\section{Secondary flows in a turbulent square duct}

Attention shifts now to the problem of corner vortices in a turbulent square duct. Previous efforts, based on kinetic energy budgets and vorticity balances, aimed at revealing the mechanism of creation of such vortices met with mixed success. In the spirit of the approach by Crighton \& Gaster (1976) we model the large-scale coherent structures as instability 'modes' of the mean velocity profile. Hence, we perform a triple decomposition of the field, like Reau \& Tumin (2002), as follows:

$$
\begin{array}{r}
(u, v, w, p)=[U(y, z), 0,0, P(x)]+[\tilde{u}(x, y, z), \tilde{v}(x, y, z), \tilde{w}(x, y, z), \tilde{p}(y, z)] \\
+\left[u^{\prime}, v^{\prime}, w^{\prime}, p^{\prime}\right](x, y, z, t) .
\end{array}
$$

The first term on the right of (3.1) represents the steady, fully developed streamwise flow $U(y, z)$, driven by the mean pressure gradient $\mathrm{d} P / \mathrm{d} x$. The steady contribution $(\tilde{u}, \tilde{v}, \tilde{w}, \tilde{p})$ represents the large-scale secondary flows, and the last term $\left(u^{\prime}, v^{\prime}, w^{\prime}, p^{\prime}\right)$ models the incoherent, fine-scale fluctuations. For the same flow, a similar decomposition (with the first two terms merged) was made by Demuren \& Rodi (1984), who arrived at the same parabolic system of equations, which will be derived below. They focused on the developing flow starting from a uniform streamwise velocity condition at the inlet (with no cross-flow), up the fully developed 


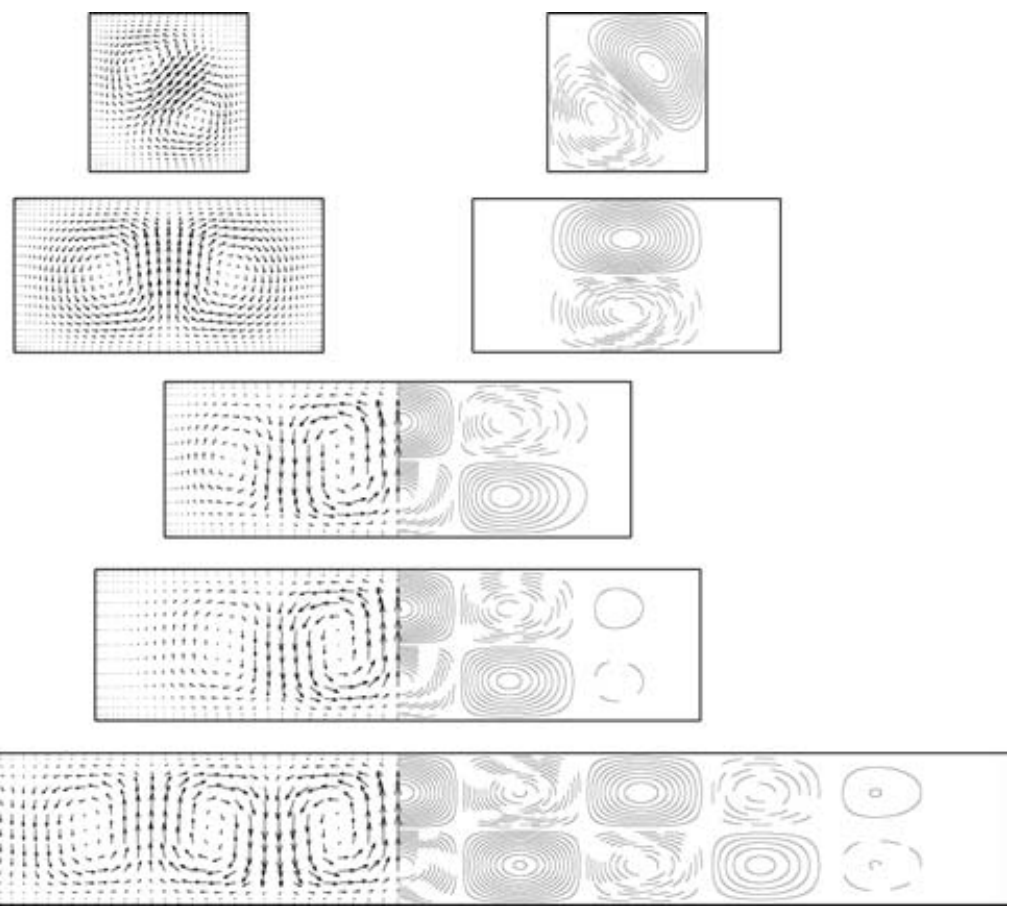

FIGURE 2. Optimal perturbations (secondary velocity vector plots on the left) and isolines of the streamwise disturbance velocity at $x_{\text {opt }}$ for $A=1-4$ and $A=8$. Positive values of $u$ are plotted with solid lines. For $A \geqslant 3$ the results show half a domain; they are symmetric about the vertical centreline $y=0$.

(a)

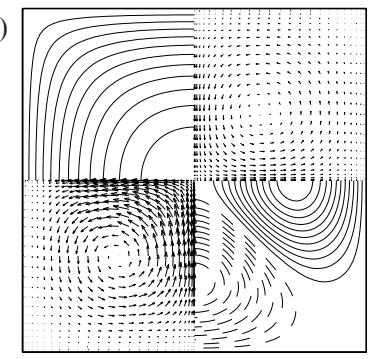

(b)

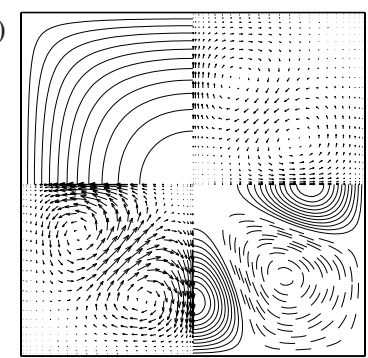

FiguRE 3. Solutions corresponding to the second and third singular values for the flow in a duct of square cross-section. Each figure is a composite of the optimal initial condition (lower left), the ensuing streak at $x=x_{\text {opt }}$ (lower right), the final vortex at $x=x_{\text {opt }}$ (upper right), and the base flow, represented through isolines of $U$ (upper left). The velocity vectors representing the vortices at $x=0$ and $x_{\text {opt }}$ use the same scale in each figure and allow a graphical appreciation of the downstream damping of the secondary velocity components. (a) The solution pertaining to the second singular value; it displays four vortices in the cross-section and is characterized by $G_{\max } / R e^{2}=9.8 \times 10^{-5}$ at $x_{o p t}=0.0225$. The third singular value $(b)$ is such that $G_{\text {max }} / R e^{2}=4.1 \times 10^{-5}$ at $x_{\text {opt }}=0.0095$.

regime. In the present case the base flow $U(y, z)$ is a given input, and the focus is on whether $U$ can sustain the growth of secondary, large-scale structures.

The Navier-Stokes equations are rendered dimensionless by the use of the conventional scales $h, U_{0}$ and $\rho U_{0}^{2}$. Upon inserting (3.1) into the equations, neglecting streamwise diffusion, and averaging over time (this operation is denoted by overbars) 
the Reynolds-averaged equations are found:

$$
\left.\begin{array}{l}
\partial_{x} \tilde{u}+\partial_{y} \tilde{v}+\partial_{z} \tilde{w}=0, \\
U \partial_{x} \tilde{u}+\tilde{v} \partial_{y} U+\tilde{w} \partial_{z} U-\frac{1}{R e}\left(\partial_{y y} \tilde{u}+\partial_{z z} \tilde{u}\right)=-\frac{\mathrm{d} P}{\mathrm{~d} x}+\frac{1}{\operatorname{Re}}\left(\partial_{y y} U+\partial_{z z} U\right)+S_{u}, \\
U \partial_{x} \tilde{v}+\partial_{y} \tilde{p}-\frac{1}{R e}\left(\partial_{y y} \tilde{v}+\partial_{z z} \tilde{v}\right)=S_{v}, \\
U \partial_{x} \tilde{w}+\partial_{z} \tilde{p}-\frac{1}{R e}\left(\partial_{y y} \tilde{w}+\partial_{z z} \tilde{w}\right)=S_{w},
\end{array}\right\}
$$

with the advective terms

$$
\begin{aligned}
& S_{u}=-\partial_{x}\left(\tilde{u}^{2}+\overline{u^{\prime 2}}\right)-\partial_{y}\left(\tilde{u} \tilde{v}+\overline{u^{\prime} v^{\prime}}\right)-\partial_{z}\left(\tilde{u} \tilde{w}+\overline{u^{\prime} w^{\prime}}\right), \\
& S_{v}=-\partial_{x}\left(\tilde{u} \tilde{v}+\overline{u^{\prime} v^{\prime}}\right)-\partial_{y}\left(\tilde{v}^{2}+\overline{v^{\prime 2}}\right)-\partial_{z}\left(\tilde{v} \tilde{w}+\overline{v^{\prime} w^{\prime}}\right), \\
& S_{w}=-\partial_{x}\left(\tilde{u} \tilde{w}+\overline{u^{\prime} w^{\prime}}\right)-\partial_{y}\left(\tilde{v} \tilde{w}+\overline{v^{\prime} w^{\prime}}\right)-\partial_{z}\left(\tilde{w}^{2}+\overline{w^{\prime 2}}\right),
\end{aligned}
$$

neglected under the assumption that large-scale coherent motion and turbulent fluctuations are of small amplitude. Such an approximation is clearly the delicate point of the present analysis; approaches which include modelling of the Reynolds stresses have been pursued by Reau \& Tumin (2002) and Joshi \& Tumin (2004).

Low-speed streaks of turbulent boundary layers have a quite short life before the abrupt emergence of localized 'spikes' preceding the ultimate nonlinear stages of transition to turbulence. Because of the short life of such coherent structures, Butler \& Farrell (1993) conducted a temporal optimization procedure over one eddy turnover time, arguing that over such a distance turbulent fluctuations had no time to disrupt the growth of the streaks. With such a hypothesis characteristic streaks spaced close to 100 wall units apart were found, in agreement with observations. Here the aim is not to model short-lived low-speed streaks, but large-scale corner structures, whose existence and spatial persistence (Gavrilakis 1992) is tied to the presence of two orthogonal walls. Since such vortices have a long life, we search for the optimal distance, i.e. that yielding the largest gain, which is typically much longer than one eddy turnover length. The gain is defined as the ratio of final to initial energy, with the energy norm as in (2.2) (except for the absence of $R e$, because of the same scaling adopted for streamwise and cross-stream axes).

The flow distribution in the cross-section is taken from Gavrilakis' simulation: the streamwise velocity profiles along the wall bisector and along the diagonal are taken from his figure 4 . We then reconstructed the mean flow in the cross-section with a least square fit. The resulting isolines of $U$ in a cross-section are displayed in figure $4(b, c$, upper left curves) and show the presence of thin boundary layers next to the solid walls. $R e$ (based on maximum speed and half-channel height) is fixed at the value 2933 of the direct simulations and the corresponding dimensionless average pressure gradient is $-\mathrm{d} P / \mathrm{d} x=0.0052323$. The optimization proceeds as in the previous section, with the only difference that the direct system of equations is now inhomogeneous, and has the symbolic form $\boldsymbol{Q} \tilde{\boldsymbol{q}}_{x}-\boldsymbol{R} \tilde{\boldsymbol{q}}=\boldsymbol{b}$, with the vector $\boldsymbol{b}$ arising from mean flow variables (cf. (3.2)). It is important to stress here that our governing equations have been derived, and that the current study cannot be labelled as a simple stability analysis of a turbulent base profile. The result of the optimization procedure, which requires of the order of 100 iterations, is displayed in figure $4(a)$. Two oblique vortices in the cross-section extract the most energy from the mean flow. The largest distortion in streamwise speed, scaled with the peak velocity of the base 

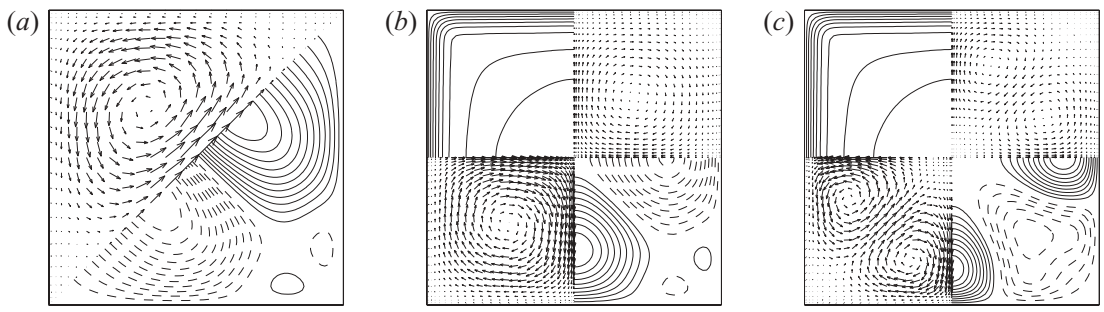

FiguRE 4. Solutions pertaining to the first three singular values of the turbulent case arranged, from left to right, in order of decreasing $G_{\max }$. (a) Secondary velocity vectors at $x=0$ and isocontours of $u$ at $x_{o p t} ;(b, c)$ as in figure 3 .

flow, ranges from 0.002 at $x=0$ to 0.109 at $x=x_{o p t}=140.78$, with a corresponding energy gain of 114.2 . This should be compared with a streamwise-average streamwise distortion close to $1 \%$ of the maximum speed indicated by Gavrilakis (1992). The length over which the initial optimal disturbance grows most corresponds to 21000 viscous units, i.e. a distance of the order of 34 large-eddy turnover units.

Clearly it would have been more suggestive to find an optimal solution made up of eight vortices in the cross-section, symmetric about the diagonals, as computed by Gavrilakis and many others. On the other hand, the present analysis suggests that a turbulent-like profile in a square duct is capable of sustaining the growth of secondary flows of a cross-stream scale comparable to that found in experiments over a distance significantly longer than the typical life span of low-speed streaks in turbulent boundary layers. To assess whether the selection of the two-vortex solution occurs sharply, a number of other calculations were conducted to identify higher singular values. The second singular value - shown in figure $4(b)$ and characterized by four vortices in the cross-section - is very close to the first $\left(G_{\max }=112.5\right.$ at $x_{\text {opt }}=83.6$ ), implying that the state corresponding to the first singular value cannot rapidly emerge out of an arbitrary initial disturbance field. The third singular value (figure $4, c$ ) exhibits a maximum gain which is about half that of the first.

The main conclusions we can draw from these calculations is that: (i) a turbulentlike mean flow is capable of supporting a variety of secondary structures; (ii) such structures can grow over long streamwise distances because of a linear transient mechanism; however, in the present simple model, they are damped downstream under viscosity; (iii) the nonlinear source terms $S_{u}, S_{v}$ and $S_{w}$ have been neglected here, but appropriate Reynolds stress modelling is indispensable for a sharp selection of the transverse field type and to achieve statistics independent of the streamwise distance $x$ (Demuren \& Rodi 1984; Mompean et al. 1996; Mompean 1998).

\section{Concluding remarks}

The first optimization of disturbances developing spatially in ducts bounded by four solid walls has been performed by a discrete direct-adjoint approach. The results for the laminar base flow complement those available in the literature and provide a possible interpretation to the experimental findings by Kao \& Park (1970) on transition in rectangular ducts. For a duct of square cross-section the maximum growth is comparable to that found in a circular pipe, and likewise for the shape of the optimal perturbation. As the aspect ratio increases, viscous diffusion from the sidewalls is less effective in hampering the growth of the vortices, so that the largest attainable gain increases, tending progressively towards the plane channel flow value.

The turbulent flow case has been approached with the assumption that large-scale structures respond linearly to the mean shear, an idea which has circulated for quite 
some time and in different guises. The result of the optimization shows that, in a square duct, the two-vortex and the four-vortex solutions are equally capable of extracting energy from a mean flow with a turbulent-like profile, over a streamwise length of several thousand viscous units. This finding seems to imply that nonlinearities are not necessary for the sustainment of secondary flows. Conversely, appropriate modelling of the turbulent stresses is needed to select key features of such secondary structures, such as their symmetry, and to ensure $x$-independent statistics.

\section{REFERENCES}

Biau, D. \& Bottaro, A. 2004 Transient growth and minimal defects: Two possible initial paths of transition to turbulence in plane shear flows. Phys. Fluids (in press).

BrundretT, E. \& BAINES, W. D. 1964 The production and diffusion of vorticity in duct flow. J. Fluid Mech. 19, 375-394.

ButLer, K. M. \& FARrell, B. F. 1993 Optimal perturbations and streak spacing in wall-bounded turbulent shear flow. Phys. Fluids A 5, 774-777.

Canuto, C., Hussaini, M. Y., Quarteroni, A. \& Zang, T. A. 1988 Spectral Methods in Fluid Dynamics. Springer.

Crighton, D. G. \& Gaster, M. 1976 Stability of slowly diverging jet flow. J. Fluid Mech. 77, 397-413.

Demuren, A. O. \& Rodi, W. 1984 Calculation of turbulence-driven secondary motion in non-circular ducts. J. Fluid Mech. 140, 189-222.

FArrell, B. F. 1988 Optimal excitation of perturbations in viscous shear flow. Phys. Fluids 31, 2093-2102.

GaVRILAKIS, S. 1992 Numerical simulation of low Reynolds-number turbulent flow through a straight square duct. J. Fluid Mech. 244, 101-129.

Gessner, F. B. 1973 The origin of secondary flow in turbulent flow along a corner. J. Fluid Mech. 58, $1-25$.

Joshi, H. \& Tumin, A. 2004 Centrifugal instability in a turbulent wall jet over a circular cylinder. AIAA Paper 2004-1108.

KAO, T. W. \& PARK, C. 1970 Experimental investigations of the stability of channel flows. Part 1. Flow of a single liquid in a rectangular channel. J. Fluid Mech. 43, 145-164.

Landahl, M. T. 1990 On sublayer streaks. J. Fluid Mech. 212, 593-614.

Launder, B. E. \& YING, W. M. 1973 Prediction of flow and heat transfer in ducts of square cross-section. Proc. Inst. Mech. Engrs 187, 455-461.

LUCHINI, P. 2000 Reynolds-number-independent instability of the boundary layer over a flat surface: Optimal perturbations. J. Fluid Mech. 404, 289-309.

Matsubara, M. \& Alfredsson, P. H. 2001 Disturbance growth in boundary layers subjected to free-stream turbulence. J. Fluid Mech. 430, 149-168.

Mompean, G. 1998 Numerical simulation of a turbulent flow near a right-angled corner using the Speziale non-linear model with RNG $k-\epsilon$ equations. Computers \& Fluids 27, 847-859.

Mompean, G., Gavrilakis, S., Machiels, L. \& Deville, M. O. 1996 On predicting the turbulenceinduced secondary flows using nonlinear $k-\epsilon$ models. Phys. Fluids 8, 1856-1868.

NiKuRADSE, J. 1926 Untersuchungen über die Geschwindigkeitsverteilung in turbulenten Strömungen. PhD Thesis, University of Göttingen, Germany.

Reau, N. \& Tumin, A. 2002 On harmonic perturbations in a turbulent mixing layer. Eur. J. Mech. B/Fluids 21, 143-155.

Reshotкo, E. \& Tumin, A. 2001 Spatial theory of optimal disturbances in a circular pipe flow. Phys. Fluids 13, 991-996.

Robitaillié-Montané, C. \& Casalis, G. 2003 Méthode de collocation spectrale appliquée à un problème de stabilité donné sous forme d'équations aux derivées partielles. Onera Tech. Rep. RT 1/07895 DMAE, Toulouse, France.

Schmid, P. J. \& Henningson, D. S. 2001 Stability and Transition in Shear Flows. Springer.

Tatsumi, T. \& Yoshimura, T. 1990 Stability of the laminar flow in a rectangular duct. J. Fluid Mech. 212, 437-449. 